\title{
A Review on Computer - Aided Formulation Development
}

\author{
Chaudhari R. G. *, Raut D. B., Barhewar P. A., Mali A. S., Burade K. B.
}

Department of Pharmaceutics, Government College of Pharmacy Karad, Satara, Maharashtra, India

\begin{abstract}
This chapter introduces the concept of formulation development assisted by computer applications. Development and optimization of various types of pharmaceutical emulsions microemulsions, selfmicroemulsifying systems, and double emulsions are presented. Illustrative examples are presented to demonstrate the ability of computer-aided tools to facilitate formulation development. Various techniques, such as design of experiments and artificial neural networks, are implemented for optimization of the formulation and/or processing parameters. Furthermore, some of the critical quality attributes and processing parameters are optimized simultaneously. The examples presented should serve as the foundation for the future qualityby-design development of pharmaceutical emulsion and (self) microemulsion formulations.
\end{abstract}

Keywords : Pharmaceutical Emulsions, Microemulsions, Self-Microemulsifying Drug Delivery Systems (SMEDDS), Double Emulsions, Formulation, Optimization.

\section{INTRODUCTION}

This chapter introduces the concept of formulation development assisted by computer applications. Due to their complex composition, preparation and stability issues of emulsions were selected to showcase various computer- aided tools in pharmaceutical formulation development. Successful development of an emulsion formulation is dependent on both formulation ingredients and processing parameters, which is especially signifi cant for more complex formulation types, such as self- emulsifying systems or double emulsions. The examples provided illustrate techniques used to defi ne a design space, select the appropriate formulation ingredient, and optimize the formulation composition as well as process parameters, according to the quality- by-design (QbD) concept. Importantly, methods that allow simultaneous optimization of multiple factors are also presented. The following chapters will provide a deeper insight into selected in silico methods.

\section{Application of computer- aided techniques in development of pharmaceutical emulsions}

Emulsions are dispersing systems made of two immiscible liquids. One liquid is dispersed into the other, in the presence of surface-active agents, such as emulsifier(s). The two immiscible liquids are usually oil and water, and the main types of simple emulsions are oil- in-water $(\mathrm{o} / \mathrm{w})$ or water- in-oil (w/o). In the pharmacy, emulsions have a great potential as vehicles for active ingredients for different routes of administration (topical, parenteral, oral). However, emulsions are thermodynamically unstable systems, and different phenomena during storage could occur, including gravitational separation (creaming/sedimentation), flocculation, coalescence, Ostwald ripening, and phase inversion.

Stability and properties of emulsions are influenced by different factors. Formulation and process optimization techniques would be useful for finding 
the ideal emulsion formulation. The main parameters relating to the stability, effectiveness, and safety of the pharmaceutical emulsion should be optimized simultaneously.

More intense application of different in silico techniques in process and formulation optimization started at the end of the last century (Gašperlin et al., 1998, 2000; Prinderre et al., 1998; Simovic et al., 1999).

Prinderre et al. (1998) applied factorial design methods to optimize the stability and suggested the required hydrophilic lipophilic balance (HLB) of o/w emulsions prepared with sodium lauryl sulfate as the surfactant. The independent variables and their levels (low/high) were mixing rate (rpm) (500/900), homogenization (no/yes), and mixing time (min) (10/20). Dependent variables were the average size of the droplets, the emulsion viscosity, and the conductivity. Experimental design determined the required HLB with good approximation in five runs for the average diameter and viscosity studies, while the conductivity study needed at least eight runs.

Similarly, Simovic et al. (1999) investigated the influence of the processing variables on performance of $\mathrm{o} / \mathrm{w}$ emulsion gels stabilized by a polymeric emulsifier (Pemulen ${ }^{\circledR}$ TR-2NF). A two-f actor threelevel experimental design at two sets was applied: using a laboratory mixer and a disperser. Independent variables were mixing speed and time, whereas dependent variables were millimeters of oil phase separated after centrifugation at $3500 \mathrm{rpm}$ in a laboratory centrifuge, and viscosity at shear rate of 180 l/s. The responses were fitted into a second- order model by means of multiple regression analysis. The authors (Simovic et al., 1999) could defi ne the most favorable conditions for preparing stable $\mathrm{o} / \mathrm{w}$ emulsions, using the laboratory mixer, with a mixing speed at $1500 \mathrm{rpm}$ and mixing time of 20 minutes.
Rahali et al. (2009) found the optimal preservative combination and concentration for preparing topical emulsions by using a D-optimal experimental design (mixture design). In this study, three preservatives were tested, benzoic acid, sorbic acid, and benzyl alcohol. The preservative effects were evaluated using the antimicrobial preservative efficacy test (challenge test) of the European Pharmacopeia (EP). The results of this study were analyzed with the help of Design Expert ${ }^{\circledR}$ software. The authors (Rahali et al., 2009) were able to formulate topical emulsions in accordance with the requirements of the EP.

Simultaneous study of the influences of different factors for emulsion systems is difficult, due to the problems of complicated nonlinear correlations. The artificial neural networks (ANN) technique seems to provide a useful tool for solving these problems. Gašperlin et al. (1998) investigated the influence of different ratios of individual components on the viscoelastic behavior of semisolid lipophilic emulsion systems using this technique. The creams were prepared according to a preliminary experimental design (mixture design). ANN involved 3 input, 12 hidden, and 9 output neurons. The input neurons were the contents of the particular emulsion components (silicone surfactant Abil ${ }^{\circ}$ WE 09, purifi ed water, white petrolatum) and the output neurons were the measured values of dynamic rheological parameter, $\tan \delta$, at different time intervals. They could be predicted using a neural network model and the results have shown a great degree of reliability. Similarly, a neural network model could be used for prediction of the complex dynamic viscosity of these semi- solid w/o emulsions (Gašperlin et al., 2000).

Other authors (Kumar et al., 2011) applied the ANN model to optimize the fatty alcohol concentration in the formulation of an o/w emulsion. Emulsion was composed of purifi ed water, liquid paraffin, sodium lauryl sulfate, and fatty lauryl alcohol. Predictions 
from ANNs are accurate and allow quantification of the relative importance of the inputs. Furthermore, by varying the network topology and parameters, it was possible to obtain output values (zeta potential, viscosity, conductivity, and particle size) that were close to experimental values. The ANN model predicted results and the actual output values were compared. An R 2 value of 0.84 for the model suggested adequate modeling, which is supported by the correlation coeffi cient value of 0.9445 .

Multiple (or double) emulsions are even more complex dispersion systems, also known as 'emulsions of emulsions'. The most common multiple emulsions are of the w/o/w type, although, for some specific applications, o/w/o emulsions can also be prepared. Usually, the multiple emulsions have been produced in a two-s tep-production process: the fi rst one for production of the primary emulsion, and the second for production of the multiple emulsions.

In w/o/w emulsions, oil globules, containing small droplets of water, are dispersed in an aqueous continuous phase. The advantages of these types of emulsion systems are relatively high entrapment capacity for hydrophilic compounds, protection of the encapsulated substances towards degradation, the ability to introduce incompatible substances into the same system, and sustained release of active substance. These characteristics make them potentially interesting for application in pharmaceutics and cosmetics. However, in practice, significant problems may arise because of their thermodynamic instability and strong tendency for coalescence, flocculation, and creaming. The stability of $\mathrm{w} / \mathrm{o} / \mathrm{w}$ emulsion may be affected by a number of factors, including the method of preparation, osmotic balance between the internal and external water phase, phase volumes ratio, type, and concentration of the emulsifiers.
In the work of Onuki et al. (2004), formulation optimization of the w/o/w multiple emulsion incorporating insulin was performed, based on statistical methods such as the orthogonal experimental design and the response surface evaluation. As model formulations, 16 types of emulsions were prepared according to the orthogonal experimental design. To optimize the formulation, the influences of five factors on characteristics of the emulsion were evaluated first. Inputs were amounts of gelatin, insulin, oleic acid, volume ratio of the outer water phase, and agitation time of the second emulsification process. Outputs were inner droplet size, viscosity, stability, and pharmacological effect. Based on Analysis of Variance (ANOVA), it was concluded that the most predominant contribution of all causal factors was the volume ratio of the outer water phase. As for the optimization study, the optimum formulation with respect to pharmacological hypoglycemic effect in rats and stability of emulsion was estimated using a simultaneous optimization technique, in which a multivariate spline interpolation (MSI) was incorporated. A two- factor composite second-order spherical experimental design was used to select model formulation. The data measured for the model formulations were analyzed by a computer program (dataNESIA, Yamatake, Tokyo, Japan). The authors (Onuki et al., 2004) reported that the optimum formulation had pharmacological activity and stability as high as a pharmaceutical formulation.

The second emulsification step could be critical for the production of multiple emulsions. For this reason, Lindenstruth and Müller (2004) examined the second emulsification step in the formulation of $\mathrm{w} / \mathrm{o} / \mathrm{w}$ multiple emulsions. Unvaried primary w/o emulsion, with diclofenac sodium as the active ingredient in the inner water phase, was used during the investigation. In the second step, a central composite design was used, and the process parameters pressure and 
temperature were varied. The multiple droplet size and the encapsulation rate of totally 10 emulsions were determined after 1,3 , and 5 homogenization cycles, to investigate the influence of process parameters. For statistical analysis, the Statistica ${ }^{\circledR}$ program was used. It was shown that the pressure and temperature, as process parameters in the second step, influened the size of multiple droplets in the $\mathrm{w} / \mathrm{o} / \mathrm{w}$ multiple emulsion. Further experiments with different w/o emulsions resulted in w/o/w multiple emulsions with different encapsulation rates of diclofenac sodium.

In the work of Wei et al. (2008), formulation optimization of emulsifiers for preparing $\mathrm{w} / \mathrm{o} / \mathrm{w}$ multiple emulsions was performed in respect of stability by using the ANN technique. The emulsifiers used were sorbitan monooleate (Span 80) and polysorbate 80 (Tween 80 ). The stability of multiple emulsions was expressed by the percentage of reserved emulsion volume of freshly prepared sample after centrifugation. Individual properties of multiple emulsions, such as droplet size, phase angle $\delta$, viscosity of the primary, and the multiple emulsions were also considered.

A back propagation (BP) network was well trained by experimental data pairs and then used as an interpolating function to estimate the stability of emulsions of different formulations. It was found that multiple w/o/w emulsions could be prepared by using mixtures of Span 80 and Tween 80 with different mass ratio as both lipophilic and hydrophilic emulsifiers. The stability is sensitive to the mixed HLB numbers and concentration of the emulsifiers. By feeding the ANN with 39 pairs of experimental data, the ANN was well trained and could predict the influences of several formulation variables on the immediate emulsions stability. The validation test indicated that the immediate stability of the emulsions predicted by the ANN was in good agreement with measured values. The ANN therefore could be a powerful tool for rapid screening for emulsifier formulation.

Different optimization techniques, for example experimental design using response surface modeling or ANN method, could be used to determine the optimal cosmetic formulations, such as depilatory cream or an $\mathrm{o} / \mathrm{w}$ emulsion vehicle for a permanent hair dye (Moulai Mostefa et al., 2006; Balfagon et al., 2010).

\section{Application of computer - aided techniques in development of microemulsion drug carriers}

Microemulsions are thermodynamically stable and optically isotropic transparent colloidal systems consisting of water, oil, and surfactant. Although they are clear, low viscous liquids, the different types of microstructures are identified (i.e. w/o, o/w, and bicontinuous), all organized on the level below 100 $\mathrm{nm}$. The microstructure of microemulsions is determined by physicochemical properties and concentrations of the constituents. Such unique systems, as well as their water- free preconcentrates, so- called self- microemulsifying oil/surfactant mixtures, are of increasing interest as potential drug delivery vehicles with long- term stability, considerable capacity for drug solubilization, and great potential for bioavailability enhancement (Fanun, 2009). Development of such carriers requires a complex strategy balancing all relevant aspects. In systems consisting of water, oil, and tenside(s), a diverse range of colloidal systems and coarse dispersions may form (e.g. emulsions, microemulsions, micelles, lyotropic liquid crystals), depending on physicochemical properties and quantitative ratios of constituents and temperature. Microemulsions and self- microemulsifying drug delivery systems (SMEDDS) form only in well balanced mixtures of the selected excipients and within the specific 
concentration ranges of the constituents at given temperatures and pressures (i.e. the microemulsion area). The analysis of the influence of formulation variables on the area of microemulsion systems is usually performed within the phase behavior studies. Pharmaceutically applicable microemulsions consist of five (surfactant, cosurfactant, oil, water, and drug) or more components. Complete phase behavior differentiation in such multicomponent mixtures requires a large number of experiments (Alany et al., 2009; Friberg and Aikens, 2009). Furthermore, characterization of a microstructure is a diffi cult task, due to its dynamic character as well as nanoscale organization (Tondre, 2005).

ANN models were introduced as useful tools for accurate differentiation and prediction of the microemulsion area from the qualitative and quantitative composition of different microemulsion- $f$ orming systems (Richardson et al., 1996, 1997; Alany et al., 1999; Agatonovic-Kustrin and Alany, 2001; Agatonovic-Kustrin et al., 2003; Mendyk and Jachowicz, 2007; Djekic et al., 2008). The pioneer studies of Richardson et al. $(1996,1997)$ demonstrated the use of ANNs to identify the physico- chemical properties of the cosurfactant with relevance for microemulsion formation in the four-c omponent system lecithin, (surfactant)/isopropyl myristate (oil)/triple distilled water/cosurfactant. The different types of cosurfactants (i.e. short- and medium- chain alcohols, amines, acids, and ethylene glycol monoalkyl ethers) were employed. The BP feedforward algorithm of learning and four computed cosurfactant molecule properties (molecular volume (v), areas for its head group (a $\psi)$ and hydrophobe (a $\varphi$ ), and computed octanol/water $\log \mathrm{P}$ value), were selected. The output was presence $(+1)$ or absence $(-1)$ of microemulsion formation in a particular mixture.

The data required for ANN training and testing were extracted from the pseudo-ternary diagrams generated previously by Aboofazeli et al. (1994), together with the additional data from four pseudo ternary phase diagrams constructed at a fixed weight ratio of surfactant- to-cosurfactant 1:1. The trained ANN (the in- house software YANNI) with the final architecture involving 6 input neurons, a single hidden layer of 14 neurons, and 1 output neuron, was shown to be highly successful in predicting phase behavior for the investigated systems from the computed values of $\mathrm{v}, \mathrm{a} \psi$, a $\varphi$, and $\log \mathrm{P}$, achieving mean success rates of 96.7 and $91.6 \%$ for training and test data, respectively. These investigations pointed to the potential of the trained ANN to screen out cosurfactants considering only the molecule features and gave an idea for more general networks, trained with data on systems involving other oils, surfactants, and surfactant- to-cosurfactant ratios. In a related study, Agatonovic-Kustrin and Alany (2001) estimated the influence of the cosurfactant on phase behavior of the fi ve- component systems (ethyl oleate (oil)/a mixture of sorbitan monolaurate and polyoxyethylene 20 sorbitan monooleate (surfactant+cosurfactant)/deionized water/n- alcohols (1-propanol, 1-butanol, 1-hexanol, and 1-octanol) or 1,2-alkanediols (1,2-propandiol, 1,2-pentanediol, 1,2hexanediol, and 1,2-octanediol)(cosurfactant)). A supervised network with a multilayer perceptron (MLP) architecture with a BP learning rule (Neural Networks ${ }^{\circledR}$, StatSoft Inc, Tulsa, USA), was used to correlate phase behavior of the investigated systems with cosurfactant descriptors (inputs), which were preselected by a genetic algorithm (GA) (Pallas 2.1, Compu Drug Int., San Francisco, USA and ChemSketch 3.5 freeware, ACD Inc., Toronto, Canada).

The most successful MLP ANN model, with two hidden layers comprising 14 and 9 neurons, predicted the phase behavior for a new set of cosurfactants with $82.2 \%$ accuracy for the microemulsion region. Alany et al. (1999) presented the fi rst report describing the utility of ANNs in predicting phase behavior of the 
four component system (ethyl oleate (oil)/sorbitan monolaurate (primary surfactant)/ polyoxyethylene 20 sorbitan monooleate (secondary surfactant)/ deionized water) regarding the components ratio. The BP training algorithm was selected. The training and testing data were extracted from several pseudoternary triangles, which represented the cuts through the phase tetrahedron. The inputs were percentages of oil and water and HLB values of the surfactants blend. The outputs were the corresponding systems (o/w emulsion, w/o emulsion, microemulsion, and liquid crystals). The trained MLP (ANNs simulator software, NNMODEL Version 1.404, Neural Fusion), with 1 hidden neuron, was tested on validation data and an accuracy of 85.2 to $92.9 \%$ was estimated, depending on the output critical values used for the classification. The low error rate demonstrated the success in employing ANNs to predict phase behavior of quaternary systems.

The fundamental goal in SMEDDS development is to optimize the surfactant/cosurfactant/oil mixture, in order to achieve sufficient drug solubility and infinite dilutability with water phase. However, there is a risk of disturbing the thermodynamic stability on dilution with the subsequent drug precipitation (Kyatanwar et al., 2010). The study of Mendyik and Jachowicz (2006) describes the development of the system of 11 ANN models suitable for further prediction of phase behavior of microemulsion- forming systems analyzing only the properties and contents of the components. The ANN models were developed by digitalization of phase diagrams of 118 systems, published in the relevant literature. The inputs were the concentrations of the constituents (surfactant, cosurfactant, oil, water) and their molecule features (ionic strength of the water phase, HLB value of the surfactant, density of the oil, etc.). The outputs confirmed whether the microemulsion is present or not for a particular quantitative and qualitative composition. The ANN based optimization technique was extended with Neuro-Fuzzy Modeling (NFM). There have been varied numbers of hidden layers of the MLP network (from 1 to 5) and up to 100 nodes in neuro-fuzzy systems, by using neural networks simulator Nets2004 written by the authors. After the architecture search step, 10 of the best ANNs were selected to become the expert committee . In addition, a so- called second- order ANN was employed to combine outputs of the abovementioned ANNs and to produce the fi nal decision of the system. The developed expert system was applied in selection of the surfactant/cosurfactant/water mixtures (microemulsion preconcentrates) with the high capacity for water solubilization. The performance of the system was estimated on $77 \%$ of properly classified data records.

Novel investigations pointed to the significance of biocompatible SMEDDS with the reduced risk for drug precipitation on water dilution, stabilized with the nonionic surfactants of polyglycolized glyceride types such as Labrasol $\AA$ and Labrafi $1 \AA$, Gelucire ${ }^{\circledR}$, Cremophor ${ }^{\circledR}$, and Plurol ${ }^{\circledR}$ groups (Fanun, 2011). Djekic et al., (2011) evaluated the influence of the cosurfactant type, the relative content of the cosurfactant (expressed as a surfactant- tocosurfactant mass ratio $(\mathrm{K} \mathrm{m})$ ), and the oil phase content (expressed as an oil-t osurfactant/cosurfactant mixture mass ratio $(\mathrm{O} / \mathrm{SCoS}))$ on the water solubilization capacity (W $\max , \% \mathrm{w} / \mathrm{w}$ ) of Labrasol ${ }^{\circledR} \quad$ (PEG-8 $\quad$ caprylic/capric glycerides)/cosurfactant/isopropyl myristate/water, by application of ANN modeling. The cosurfactants were commercial nonionic tensides: Plurol ${ }^{\circledR}$ Isostearique (polyglyceryl-6 isostearate); Cremophor ${ }^{\circledR}$ RH40 (PEG-40 hydrogenated castor); Solubilisant gamma ${ }^{\circledR}$ 2421 ((Octoxynol-12 (and) Polysorbate 20)); and Solubilisant gamma ${ }^{\circledR} 2429$ (Octoxynol-12 (and) Polysorbate 20 (and) PEG-40 Hydrogenated castor oil). The $\mathrm{K} \mathrm{m}$ values were $4: 6,5: 5$, and $6: 4$. The $\mathrm{SCoS} / \mathrm{O}$ values were varied from 1:9 to 9:1. The water 
solubilization limit was detected by titrating the $\mathrm{O} / \mathrm{SCoS}$ mixtures with water. The results were used to generate the inputs and output for ANN training. The inputs were $\mathrm{K} \mathrm{m}$ and $\mathrm{SCoS} / \mathrm{O}$ values. The output was the water solubilization limit ( $\mathrm{W} \max , \% \mathrm{w} / \mathrm{w})$. The appropriate selection of network architecture was the milestone in utilization of ANNs. A Generalized Regression Neural Network (GRNN), MLP, and Radial Basis Function (RBF) ANN architectures (Statistical Neural Networks, StatSoft, Inc., Tulsa, OK, USA) were used throughout the study. In the presence of the Plurol ${ }^{\circledR}$ Isostearique cosurfactant, a feed-forward GRNN comprising four layers (the fi rst layer had 2 input units, the second layer had 27 hidden units, the third layer had 2 units, and the fourth layer had 1 output unit), was characterized by the generalization ability of $99.1 \%$. When Cremophor ${ }^{\circledR}$ RH40 was used as a cosurfactant, a MLP network with 4 layers was generated with the prediction ability of $92 \%$ for training data set, $93 \%$ for validation data set, and $92 \%$ for test data set. In systems with Solubilisant gamma ${ }^{\circledR} \quad 2421$ and Solubilisant gamma ${ }^{\circledR} 2429$, satisfactory results were achieved with the RBF network. The ANN models provided a deeper understanding and prediction of a water solubilization limit for any combination of surfactant concentration and oil concentration in their mixture, within the investigated range. Learned networks were used for modeling, simulation, and optimization of the microemulsion area boundary by testing experimental points in experimental fields; searching for the optimal solutions; and presenting response surfaces (or contour plots). Response surfaces presenting the infl uence of the surfactant concentration in the surfactant/cosurfactant mixture and the oil concentration in the mixture with tensides on the water solubilization limit, pointed to the maximum performance in the presence of Cremophor® ${ }^{\circledR} H 40$ at high $\mathrm{SCoS} / \mathrm{O}$ ratios ( $\mathrm{SCoS} / \mathrm{O}>7: 3$ ) within the investigated $\mathrm{K} \mathrm{m}$ range. Such mixtures would be the most promising regarding the self- microemulsification phenomenon. The combination of the titration method for phase behavior data collection with in silico data modeling, demonstrated in this study, is a particularly useful approach in development of SMEDDS, which allows to follow dilution of self-microemulsifying concentrate with the aqueous phase in a continuous manner.

The study of Podlogar et al. (2008) demonstrated that ANN modeling could be effective in minimizing the experimental efforts characterizing complex structural features of microemulsions. Two evolutionary ANNs (Yao, 1991) have been constructed by introducing GA to the feed-forward ANN, one being able to predict the type of microemulsion from its composition and the second to predict the type of microemulsion from the differential scanning calorimetry (DSC) curve. The components of the microemulsion- forming system were isopropyl myristate (oil), polyoxyethylene (20) sorbitan monopalmitate (Tween ${ }^{\circledR} 40$ ) (surfactant), glyceryl caprylate (Imwitor $\left.{ }^{\circledR} 308\right)$ (cosurfactant), and twice distilled water. The type of microemulsion microstructure (i.e. o/w, bi- continuous, w/o) was differentiated by measuring the freezing peak of the water in DSC thermograms. The data pool used to train both ANNs included the composition of 170 microemulsion samples and DSC curves. To determine the type of microemulsion from its composition, a feed- forward network was programmed, with the fi nal architecture involving 4 input neurons, a single hidden layer of 12 neurons, and 5 output neurons. To determine the type of microemulsion from its DSC curve, a second feed-f orward ANN with 1 hidden layer was constructed, containing 100 input neurons, a single layer of 5 hidden neurons, and 5 output neurons. Both ANNs showed an accuracy of $90 \%$ in predicting the type of microemulsion from the previously untested compositions. 


\section{IV.CONCLUSION}

A nonlinear mathematical approach comprising experimental design, neural networks, GAs, and/or neuro-fuzzy logic represents a promising tool for in silico modeling of formulation procedures in development of emulsion and (micro)emulsion drug carriers. Although in silico formulation is not a substitute for laboratory experiments, the results of current efforts clearly demonstrated a potential to shorten the time necessary to fi nd optimal quantitative and qualitative composition. Also, this strategy is capable of generating new potential (micro)emulsion forming systems. The upcoming step would be application of such methodology as a tool to correlate composition/structure characteristics with the biopharmaceutical profiles of (micro)emulsion drug delivery systems, which is encouraging for their future development.

\section{REFERENCES}

[1]. Aboofazeli, R., Lawrence, C.B., Wicks, S.R., and Lawrence, M.J. ( 1994 ) 'Investigations into the formation and characterization of phospholipid microemulsions. III: Pseudo- ternary phase diagrams of systems containing water- lecithinisopropyl myristate and either an alkanoic acid, amine, alkanediol, polyethylene glycol alkyl ether or alcohol as cosurfactant', Int. J. Pharm., $111: 63-72$.

[2]. Agatonovic-Kustrin, S. and Alany, R.G. ( 2001 ) 'Role of genetic algorithms and artifi cial neural networks in predicting the phase behavior of colloidal delivery systems ', Pharm. Res., 18 ( 7 ): $1049-55$.

[3]. Agatonovic-Kustrin, S., Glass, B.D., Wisch, M.H., and Alany, R.G. ( 2003 ) 'Prediction of stable microemulsion formulation for the oral delivery of a combination of anti- tubercular drugs using ANN methodology ',Pharm. Res., 20 : 1760 - 4 .

[4]. Alany, R.G., Agatonovic-Kustrin., Rades.T., and Tucker, I.G. ( 1999 ) 'Use of artificial neural networks to predict quaternery phase systems from limited experimental data ', J. Pharm. Biomed.,19: 444 -52 .

[5]. Alany, R.G., El Maghraby,G.M.M., KrauelGoellner,K., and Graf, A.( 2009 ) 'Microemulsion systems and their potential as drug carriers ', in M. Fanun (ed.) Microemulsions: Properties and Applications, pp. 247 - 91 .

[6]. Boca Raton, FL : CRC Press, Taylor \& Francis Group . Balfagón, A.C.,Serrano-Hernanz, A.,Teixido,J.,and Tejedor-Estrada, R.( 2010 ) 'Comparative study of neural networks and least mean square algorithm applied to the optimization of cosmetic formulations ', Int. J. Cosmet. Sci., 32 : 376 - 86 .

[7]. Djekic, L., Ibric, S., and Primorac, M. ( 2008 ) 'The application of artificial neural networks in the prediction of microemulsion phase boundaries in PEG-8 caprylic/capric glycerides based systems ', Int. J. Pharm., 361 ( 1 - 2 ): 41 6 .

[8]. Djekic, L., Ibric, S., and Primorac, M. ( 2011 ) 'Application of artificial neural networks (ANNs) in development of pharmaceutical microemulsions ', in J.A. Flores (ed.) Focus on Artificial Neural Networks, pp. 1 - 28 . New York : Nova Science Publishers . Fanun, M. ( 2011 ) 'Biocompatible microemulsions', in M. Fanun, (ed.) Colloids in Biotechnology, pp. 417 -36 .

[9]. Boca Raton, FL: C RC Press, Taylor \& Francis Group .Fanun, M. (ed.) ( 2009 ) Microemulsions: Properties and Applications . Boca Raton, FL : CRC Press, Taylor \& Francis Group . Friberg, S.E. and Aikens, P.A. ( 2009 )'A phase diagram approach to microemulsions ', in 
M. Fanun, (ed.) Microemulsions: Properties and Applications, pp. 1 - 15 .

[10]. Boca Raton, FL: CRC Press, Taylor \& Francis Group . Gašperlin, M. Tušar, L. Tušar, M. Kristl, J., and Šmid-Korbar, J. ( 1998 ) 'Lipophilic semisolid emulsion systems: viscoelastic behaviour and prediction of physical stability by neural network modeling', Int. J. Pharm., 168 : 243- 54 .

[11]. Gašperlin, M., Tušar, L. Tušar, M., Šmid-Korbar, J., Zupan, J., and Kristl, J. ( 2000 ) 'Viscosity prediction of lipophilic semisolid emulsion systems by neural network modelling ', Int. J. Pharm., 196 : 37 - 50 .

[12]. Kumar, K.J., Panpalia, G.M., and Priyadarshini, S. ( 2011 ) 'Application of artificial neural networks in optimizing the fatty alcohol concentration in the formulation of an $\mathrm{o} / \mathrm{w}$ emulsion ', Acta Pharm., 61 : 249 - 56 . Kyatanwar, A.U., Jadhav, K.R., and Kadam, V.J. ( 2010 ) 'S elf micro- emulsifying drug delivery system (SMEDDS): Review', J. Pharm. Res., 3 ( 1 ): $75-83$.

[13]. Lindenstruth, K. and Müller, B.W. ( 2004 ) 'W/O/W multiple emulsions with diclofenac sodium ', Eur. J. Pharm. Biopharm., 58 : 612 - 27

[14]. Mendyk, A. and Jachowicz, R. ( 2006 ) 'ME_expert - a neural decision support system as a tool in the formulation of microemulsions',Biocybern. Biomed. Eng., 26 : 25 - 32 . Mendyk, A. and Jachowicz, R. ( 2007 ) 'Unifi ed methodology of neural analysis in decision support systems built for pharmaceutical technology '. Expert Syst. Appl., 32 ( 4 ): 1124 - 31 .

[15]. Moulai Mostefa,N.,Hadj Sadok, A., Sabri, N., and Hadji, A. ( 2006 ) 'Determination of optimal cream formulation from long-t erm stability investigation using a surface response modelling ', Int. J. Cosmet. Sci., 28 : 211 - 18 .
[16]. Onuki, Y., Morishita, M., and Takayama, K. ( 2004 ) 'Formulation optimization of water- inoil- water multiple emulsion for intestinal insulin delivery ', J. Control Release, 97 : 91 - 9.

[17]. Podlogar, F., Šibanc, R., and Gašperlin, M. ( 2008 ) 'Evolutionary artificial neural networks as tools for predicting the internal structure of microemulsions', J. Pharm. Pharmaceut. Sci., 11 ( 1 ): $67-76$.

[18]. Prinderre, P., Piccerelle, P., Cauture, E., Kalantzis, G., Reynier, J.P., and Joachim, J. ( 1998 ) 'Formulation and evaluation of $\mathrm{o} / \mathrm{w}$ emulsions using experimental design', Int. J. Pharm., $163: 73$ - 9 .

[19]. Rahali, Y., Pensé-Lhéritier, A.M., Mielcarek, C., and Bensouda, Y. ( 2009 ) 'Optimization of preservatives in a topical formulation using experimental design ', Int. J. Cosmet. Sci., 31 : $451-60$.

[20]. Richardson, C.J., Mbanefo, A., Aboofazeli, R., Lawrence, M.J., and Barlow, D.J. ( 1996 ) 'Neural network prediction of microemulsion phase behaviour', Eur. J. Pharm. Sci., 4 : S1 S139. Richardson, C.J., Mbanefo, A., Aboofazeli, R., Lawrence, M.J., and Barlow, D.J. ( 1997 ) 'Prediction of phase behavior in microemulsion systems using artificial neural networks ', J. Colloid. Interf. Sci., 187 ( 2 ): 296 - 303.

[21]. Simovic, S., Milic-Askrabic, J., Vuleta, G., Ibric, S., and Stupar, M. ( 1999 ) 'The influence of processing variables on performance of $\mathrm{o} / \mathrm{w}$ emulsion gels based on polymeric emulsifier (Pemulen ${ }^{\circledR}$ TR-2NF)', Int. J. Cosmet. Sci., 21 : 119 - 25 .

[22]. Tondre, C. ( 2005 ) 'Dynamic processes in microemulsions', in Z. Raoul (ed.) Dynamics of Surfactant Self-Assemblies: Micelles, Microemulsions, Vesicles and Lyotropic Phases, pp. 233 - 298 .

[23]. Boca Raton, FL: C RC Press, Taylor \& Francis Group .Wei, H.,Zhong, F., Ma, J., and Wang, Z. 
( 2008 ) 'Formula optimization of emulsifiers for preparation of multiple emulsions based on artificial neural networks ', J. Disp. Sci. Technol., 29 : 319 - 26 .

[24]. Yao, X. ( 1991 ) 'Evolution of connectionist networks ', in T. Dartnall (ed.) Reasoning and Creativity, $\mathrm{P}$ reprints Int. Symp. AI, Queensland: Australia : Griffith University .

\section{Cite this article as :}

Chaudhari R. G., Raut D. B., Barhewar P. A., Mali A. S., Burade K. B., "A Review on Computer - Aided Formulation Development", International Journal of Scientific Research in Science and Technology (IJSRST), Online ISSN : 2395-602X, Print ISSN : 23956011, Volume 6 Issue 6, pp. 328-337, NovemberDecember 2019. Available at doi : https://doi.org/10.32628/IJSRST196658 Journal URL : http://ijsrst.com/IJSRST196658 